\title{
Comparison of Hospital-Wide Code Rates and Mortality Before and After the Implementation of a Rapid Response Team
}

Muhammad Yousaf ${ }^{1}$, Sheher Bano ${ }^{1}$, Muhammad Attaur-Rehman ${ }^{1}$, Chaudhary Muhammad Junaid Nazar $^{2}$, Aayesha Qadeer ${ }^{3}$, Salma Khudaidad ${ }^{4}$, Syed Waqar Hussain ${ }^{5}$

1. Critical Care Medicine, Shifa International Hospital, Islamabad, Pakistan 2. Department of Nephrology and Renal Transplantation, Shifa International Hospital, Islamabad, Pakistan 3. Department of Pulmonology \& Critical Care Medicine, Shifa International Hospital, Islamabad, Pakistan 4. Obstetrics and Gynecology, Bolan Medical College 5. KRL Hospital Islamabad

$\square$ Corresponding author: Chaudhary Muhammad Junaid Nazar,dr.cmjnazar@live.co.uk Disclosures can be found in Additional Information at the end of the article

\section{Abstract}

\section{Objective}

To compare hospital-wide code rates and mortality before and after the implementation of a rapid response team (RRT).

\section{Study design}

A prospective cohort design with historical controls.

\section{Place of study}

This study was conducted at Shifa International Hospital, Islamabad, from January 21, 2016, to January 20, 2017.

\section{Materials and methods}

The triggers for the rapid response team (RRT) were displayed on each floor. The in-house staff was trained on when and how to activate the rapid response team (RRT). Data were collected on a specified data collection form. Mortality and hospital-wide code blue rates were calculated and compared with those from one year before the implementation of the rapid response team (RRT) (i.e., from January 21, 2015, to January 20, 2016).

Received 10/22/2017 Review began 12/26/2017 Review ended 01/08/2018 Published 01/09/2018

\section{(c) Copyright 2018}

Yousaf et al. This is an open access article distributed under the terms of the Creative Commons Attribution License CC-BY 3.0., which permits unrestricted use, distribution, and reproduction in any medium, provided the original author and source are credited.

\section{Results}

The total number of admissions during the study period was 40,177. In total, 796 RRTs were activated with a rate of activation of 19.81 per 1000 admissions. The most common activator for RRTs was an altered level of consciousness (24.12\%), followed by tachycardia (19.22\%), and tachypnea (14.45\%). The total number of admissions one year before the implementation of the RRT was 39,460. The total number of mortality events before the implementation of the RRT was 1470 (3.725\%) and after the implementation of the RRT was 1529 (3.805\%), which was not significantly different $(\mathrm{P}=.576)$. The total number of code blues before the implementation of the RRT was 146 (0.369\%) and after the implementation of RRT was 148 (0.368\%), which was not significantly different $(\mathrm{P}=.929)$. 


\section{Conclusion}

In this large single-institution study, rapid response team implementation was not associated with significant reductions in either hospital-wide code blue or mortality.

Categories: Internal Medicine, Miscellaneous, Pulmonology

Keywords: rapid response team, code blue, mortality

\section{Introduction}

In-hospital cardiac arrests continue to have a mortality rate of $80 \%$ despite advances in health care [1]. These deadly in-hospital cardiac arrests are preceded by warning signs, such as the derangements of vital signs [2], and the early detection of these warning signs may provide an opportunity to prevent cardiac arrest and its attendant mortality. Based on this, the use of a rapid response team (RRT) has been recommended as a means of reduction of in-hospital mortality [3-4]. An RRT is typically a multidisciplinary team, consisting of a physician, critical care nurse, and respiratory therapist charged with the prompt evaluation, triage, and treatment of patients with signs of clinical deterioration not treated in the intensive care unit (ICU) [5]. The team is triggered by in-house staff when certain predetermined signs of clinical deterioration are observed and can order critical laboratory and imaging studies and medications, transfer the patient to higher levels of monitoring and care, and discuss end-oflife care with the patients and their families independent of the primary physician [6].

Whether the implementation of RRTs is effective in decreasing the hospital-wide code rates and hospital mortality is unclear. Previously conducted studies have shown conflicting results. A few studies have shown that the implementation of an RRT has reduced the out of ICU cardiopulmonary arrests [7-9]. One large study [10] that included 73,000 patients in the postintervention group, as well as a few small, short-term studies [7,9], have found a significant reduction in hospital-wide mortality after RRT implementation. On the other hand, several other studies have failed to demonstrate a significant reduction in hospital-wide mortality after RRT implementation [11-12], including three of the four largest studies evaluating hospitalwide mortality, with between 25,000 and 68,000 patients in the post-intervention group [8,1112]. Moreover, a meta-analysis of 17 studies, including more than 400,000 patients, failed to find a reduction in overall hospital-wide mortality or adult mortality, although a significant reduction in pediatric mortality was observed [13].

To address these conflicting results of previous studies, we have conducted our study. Also, we wanted to know the common triggers for RRT activation in our hospital and the common interventions that the RRT team does after it is activated.

\section{Materials And Methods}

The study design was a prospective cohort with historical controls. The study was carried out at Shifa International Hospital, Islamabad, a tertiary care hospital having surgical and medical subspecialties along with liver and bone marrow transplant departments, from January 21 , 2016, to January 20, 2017. Prior to the implementation of the RRT, teaching and training sessions were carried out with all those who had to participate in the RRT system.

Signs and symptoms were displayed in the reception area of every floor, and the floor doctors and nursing staff were trained to activate the rapid response team if they notice any one of these signs and symptoms in any patient. RRT activation occurred via dedicated pagers through 


\section{Cureus}

the operator with a single emergency telephone number. The team was led by a critical care fellow and included an ICU nurse and respiratory therapist having all the necessary emergency equipment, such as a bag mask, a glucometer, and an endotracheal tube. When activated, the RRT was supposed to be present on the required floor within 10 minutes and was independent in its decision-making (e.g., advising laboratory and radiology investigations, prescribing medications, shifting the patient to a more intensively monitored area, discussing and changing the code status of the patient, continuing to manage and monitor the patient at the same place, etc.)

Data on hospital-wide mortality, code blue, and demographic characteristics were collected prospectively in the hospital core patient database and retrieved for the study. The data on the primary reason for rapid response team activation, rapid response team intervention, and patient disposition after rapid response team evaluation were prospectively collected on a data collection form by the RRT team. All patients admitted to the hospital during the duration of the study, irrespective of their age and gender, were included. Data on code blues, including location, type of arrest, and initial rhythm were similarly collected. Patients who were in procedure rooms when the RRT or code blue team was activated for them were excluded because the procedure room environment is different from the general ward environment. The hospital-wide mortality and code blue data one year before the implementation of the RRT system, from January 20, 2015, to January 20, 2016, were retrieved from the in-hospital core patient database and were compared with the mortality and code blue data collected one year after the implementation of the RRT system (i.e., from January 21, 2016, to January 21, 2017).

\section{Results}

The total number of admission before the implementation of the RRT was 39,460 and after the implementation, it was 40,177 . There was no statistically significant difference in hospitalwide mortality and code blue rates before and after the implementation of the RRT system. The total number of mortalities before the implementation of RRT was 1470 (3.725\%) and after the implementation of RRT, it was 1529 (3.805\%) with a P value of 0.576 . The total number of code blues before the implementation of RRT was 146 (0.369\%), and after the RRT implementation, the total was $148(0.368 \%)$ with a $\mathrm{P}$ value of 0.929 (Table 1 and Figure 1)

\begin{tabular}{|c|c|c|c|}
\hline Outcome & Pre-intervention & Post-intervention & $P$ value \\
\hline Admission & 39460 & 40177 & \\
\hline Mortality & $1470(3.725 \%)$ & 1529 (3.805\%) & 0.576 \\
\hline Code Blue & 146 (0.369\%) & $148(0.368 \%)$ & 0.929 \\
\hline Mean Age, Years (SD) & 42.9 (18.3) & 44 (18.2) & \\
\hline Females (Percentage) & $18388(46.6 \%)$ & $18481(46 \%)$ & \\
\hline Case MIx Index & 1.89 & 1.95 & \\
\hline
\end{tabular}

\section{TABLE 1: Summary of study outcomes before and after the implementation of the rapid response team}

Abbreviations: SD, standard deviation. 


\section{Cureus}

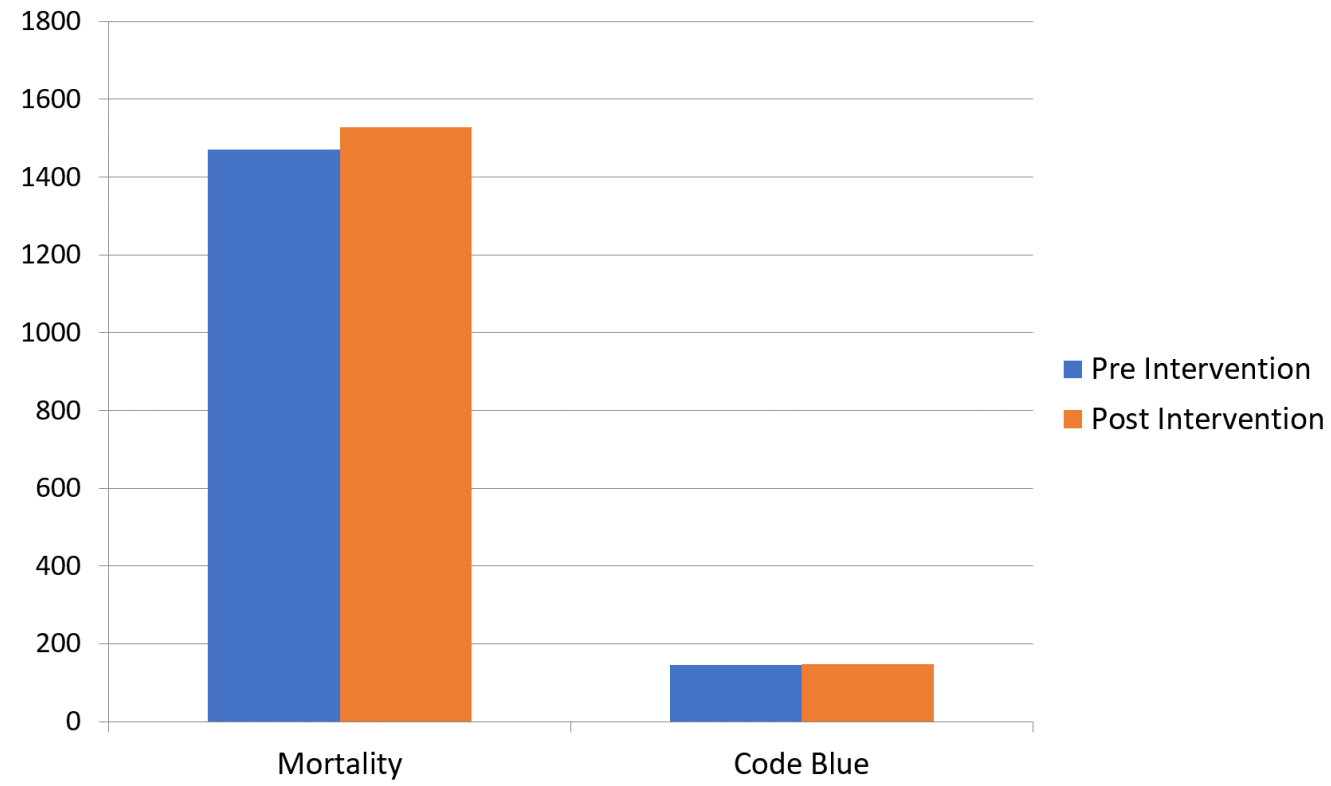

FIGURE 1: Mortality and code blue before and after the implementation of the rapid response team

In total, 796 RRTs were activated. The most common reason for RRT activation was an alteration in the level of consciousness (192 events; 24.12\%) followed by a heart rate of > 140 (153 events; 19.22\%) and tachypnea (115 events; 14.45\%). The other reasons for RRT activation were hypotension, desaturation, chest pain, uncontrolled bleeding, staff/family concerns, hypertension, bradycardia, seizures, and oliguria (Table 2). 


\section{Cureus}

\begin{tabular}{|c|c|c|}
\hline Indications for RRT activation & Number & Percentage \\
\hline Alteration in LOC & 192 & 24.12 \\
\hline Heart rate $>140 \mathrm{bpm}$ & 153 & 19.22 \\
\hline Respiratory rate $>28 \mathrm{pm}$ & 115 & 14.45 \\
\hline $\mathrm{SBP}<80 \mathrm{mmHg}$ & 92 & 11.56 \\
\hline $\mathrm{O}_{2}$ saturation $<85 \%$ & 76 & 9.55 \\
\hline Chest pain & 67 & 8.42 \\
\hline Uncontrolled bleeding & 40 & 5.03 \\
\hline Staff/family concerns & 35 & 4.40 \\
\hline $\mathrm{SBP}>180 \mathrm{mmHg}$ & 12 & 1.51 \\
\hline Heart rate $<40 \mathrm{bpm}$ & 8 & 1.01 \\
\hline Seizures & 4 & 0.50 \\
\hline Urine output $<20 \mathrm{ml} / \mathrm{hr}$ & 2 & 0.25 \\
\hline
\end{tabular}

\section{TABLE 2: Reasons for rapid response team activation}

Abbreviations: bpm, beats per minute; LOC, level of consciousness; pm, (breaths) per minute; RRT, rapid response team; SBP, systolic blood pressure.

The most common intervention taken by the RRT was supplemental oxygen (447 events; 58.13\%) followed by arterial blood gas (250 events; $32.51 \%$ ) and electrocardiogram (240 events; $31.21 \%)$. The other interventions taken by RRT were, in descending order of frequency, chest xray, cardiac enzymes, intravenous fluids, intravenous diuretics, non-invasive ventilator application, inhaled bronchodilators, airway suctioning, endotracheal intubation, intravenous steroids, anti-arrhythmic, computed tomography (CT) of the head, antihypertensives, and CT pulmonary angiogram (Figure 2). 


\section{Cureus}

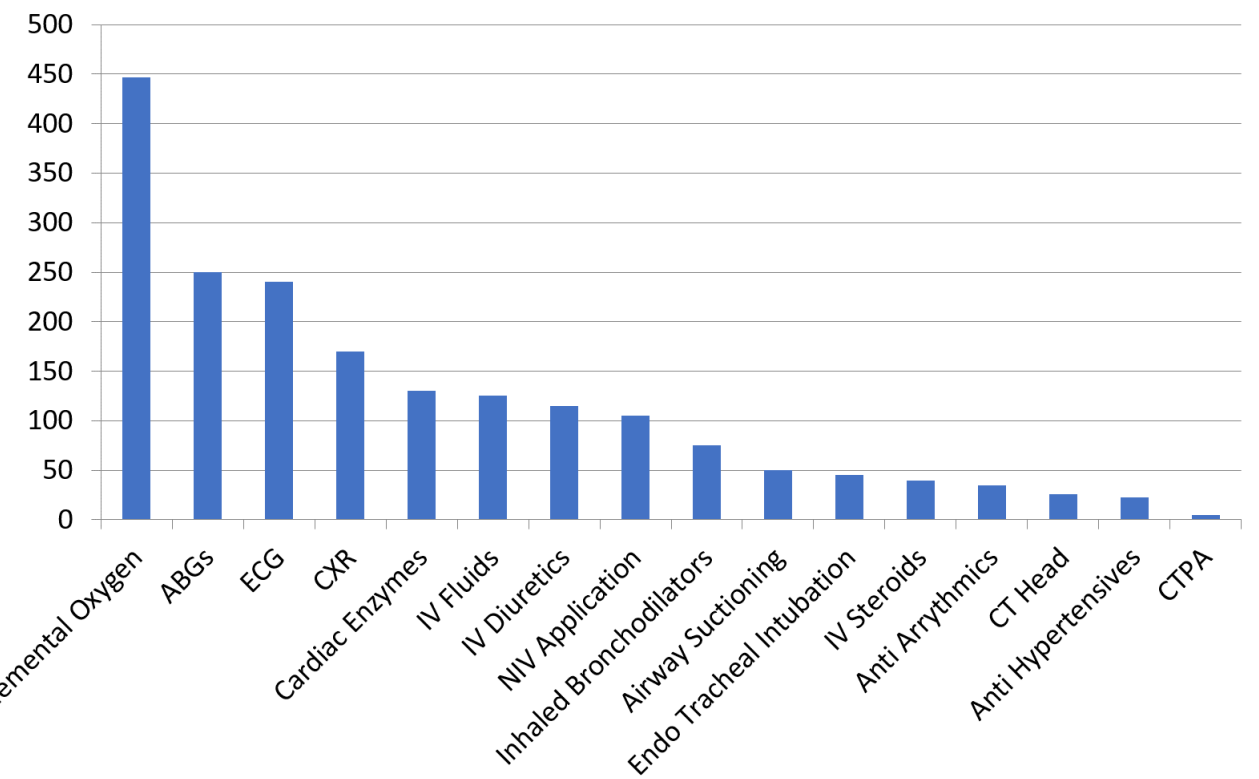

FIGURE 2: Interventions taken by the rapid response team

Abbreviations: ABGs, arterial blood gases; CT, computed tomography; CTPA, computed tomography pulmonary angiography; CXR, chest x-ray; ECG, electrocardiography; IV, intravenous; NIV, non-invasive ventilator

The disposition of patients after RRT activation is shown in Table 3.

\begin{tabular}{lllll}
\hline S.No. & Disposition Place & Number & Percentage \\
\hline 1. & HDU & 366 & 45.98 \\
\hline 2. & REMAINED ON FLOOR & 207 & 26.01 \\
\hline 3. & ICU & 107 & 13.44 \\
4. & CCU & 72 & 9.05 \\
\hline 5. & CODE STATUS CHANGED TO DNR & 40 & 5.03 \\
6. & DEATH DURING RRT & 4 & 0.50
\end{tabular}

\section{TABLE 3: Disposition of patients after rapid response team activation}

Abbreviations: CCU, coronary care unit; DNR, do not resuscitate; HDU, high dependency unit; ICU, intensive care unit; RRT, rapid response team; S.No., site number.

The 40 patients whose code status was changed to do not resuscitate remained on the floor and were excluded from the study. Of the remaining 756 patients, 636 were discharged home safely, and 120 expired during the hospital stay; the expiry site of these patients by number and percentage is shown in Table 4 


\section{Cureus}

\begin{tabular}{|c|c|c|c|}
\hline S.No. & Place of Death & Number & Percentage \\
\hline 1. & Within ICU & 50 & 41.67 \\
\hline 2. & Outside ICU & 66 & 55.00 \\
\hline 3. & During RRT & 4 & 3.33 \\
\hline
\end{tabular}

\section{TABLE 4: Site of expiry of patients during hospital stay}

Abbreviations: ICU, intensive care unit; RRT, rapid response team; S.No., site number.

\section{Discussion}

Our study compared the hospital-wide mortality and code blue rates one year before and after the implementation of the rapid response team. In addition, we evaluated the various characteristics of our rapid response team, such as the triggers of activation, the interventions taken by the rapid response team, and the disposition of the patients after rapid response team (RRT) activation. Our study included more than 40,000 patients. We have found no difference in hospital-wide mortality and code blue rates before and after the implementation of RRT. This finding is in accordance with the work of other investigators, including a large multicenter randomized controlled trial, the Medical Early Response Intervention and Therapy (MERIT) study, which did not show any improvement in code blue rates after RRT implementation [11] and a meta-analysis of 18 studies that concluded that robust evidence of the effectiveness of rapid response teams in reducing hospital mortality is lacking [13]. Although some prior studies and a meta-analysis have shown that the implementation of the RRT has decreased out-of-ICU code blue rates $[9,13]$, the extent to which this decrease is due to a reversal in physiological decline after RRT intervention or because of the transfer of the patients to the ICU by the RRT team, thus removing a potential code blue event from the study outcome because within-ICU code blues are not announced, is not known.

The code status of 40 patients out of a total of 796 patients (5.20\%) was changed to the 'do not resuscitate' status. It may be a contribution of the RRT directing hospital resources and personnel to patients who are more likely to benefit and referring those patients who need endof-life care to the palliative care department but how much this is cost-effective was not determined. Our study should be interpreted in the context of following limitations. Though it had more than 40,000 patients in the post-intervention period, it was a single institute study and included both adult and pediatric populations. Outcomes may have been different if both populations had been studied separately. All those who were involved in the activation of the RRT were trained before the implementation of the RRT on those signs and symptoms that should trigger the activation of the RRT. These were displayed in the reception area of every floor, yet, a delay in the activation of the RRT may be a factor in the lack of improvement in hospital-wide mortality and code blue rates after the implementation of the RRT. Our study was a prospective cohort study with historical controls; the two cohorts may not be identical. This is a common problem with all such studies. However, there was no epidemic in the postimplementation. As the post-implementation period was one year and was compared with the population from one year before the implementation, seasonal variation was not an issue. The hospital-wide efforts to improve the quality of patient care would have been expected to decrease the hospital-wide mortality rate and in-hospital code blue rate. However, in our study, there was no difference in mortality rate and code blue rate before and after the 
implementation of the RRT; therefore, the quality improvement effort was not a confounding factor.

\section{Conclusions}

There was no significant difference in the hospital-wide mortality rate and the in-hospital code blue rate before and after the implementation of a rapid response team in our tertiary care hospital. As our study failed to show any improvement in hospital-wide mortality rate and code blue rate after the implementation of the rapid response team, it is recommended that multicenter studies with a sufficiently long follow-up be conducted before suggesting the implementation of a rapid response team in tertiary care hospitals across the country.

\section{Additional Information Disclosures}

Human subjects: All authors have confirmed that this study did not involve human participants or tissue. Animal subjects: All authors have confirmed that this study did not involve animal subjects or tissue. Conflicts of interest: In compliance with the ICMJE uniform disclosure form, all authors declare the following: Payment/services info: All authors have declared that no financial support was received from any organization for the submitted work. Financial relationships: All authors have declared that they have no financial relationships at present or within the previous three years with any organizations that might have an interest in the submitted work. Other relationships: All authors have declared that there are no other relationships or activities that could appear to have influenced the submitted work.

\section{Acknowledgements}

We acknowledge the contribution of all critical care fellows, respiratory therapists, and ICU nurses of Shifa International Hospital in data collection, the hospital core database department for help in retrieving the data, and the in-house staff on all the floors for their cooperation. Special thanks to the hospital administration for providing logistic support for the study. No one received any compensation for their contribution.

\section{References}

1. Feingold P, Mina MJ, Burke RM, et al.: Long-term survival following in-hospital cardiac arrest: a matched cohort study. Resuscitation. 2016, 99:72-78. 10.1016/j.resuscitation.2015.12.001

2. Rozen TH, Mullane S, Kaufman M, Frank Hsiao YFF, Warrillow S, Bellomo R, Jones DA: Antecedents to cardiac arrests in a teaching hospital intensive care unit . Resuscitation. 2014, 85:411-417. 10.1016/j.resuscitation.2013.11.018

3. Berwick DM, Calkins DR, McCannon CJ, Hackbarth AD: The 100,000 lives campaign: setting a goal and a deadline for improving health care quality. JAMA. 2006, 295:324-327. 10.1001/jama.295.3.324

4. Australian Commission on Safety and Quality in Health Care: National consensus statement: essential elements for recognising and responding to clinical deterioration. 2010,

5. Devita MA, Bellomo R, Hillman K, et al.: Findings of the first consensus conference on medical emergency teams. Crit Care Med. 2006, 34:2463-2478.

10.1097/01.CCM.0000235743.38172.6E

6. Jones DA, DeVita MA, Bellomo R: Rapid-response teams. N Engl J Med. 2011, 365:139-146. 10.1056/NEJMra0910926

7. Buist MD, Moore GE, Bernard SA, Waxman BP, Anderson JN, Nguyen TV: Effects of a medical emergency team on reduction of incidence of and mortality from unexpected cardiac arrests in hospital: preliminary study. BMJ. 2002, 324:387-390. 10.1136/bmj.324.7334.387

8. Chan PS, Khalid A, Longmore LS, Berg RA, Kosiborod M, Spertus JA: Hospital-wide code rates and mortality before and after implementation of a rapid response team. JAMA. 2008, 


\section{Cureus}

300:2506-2513. 10.1001/jama.2008.715

9. Sharek PJ, Parast LM, Leong K, et al.: Effect of a rapid response team on hospital-wide mortality and code rates outside the ICU in a children's hospital. JAMA. 2007, 298:2267-2274. 10.1001/jama.298.19.2267

10. Konrad D, Jaderling G, Bell M, Granath F, Ekbom A, Martling CR: Reducing in-hospital cardiac arrests and hospital mortality by introducing a medical emergency team. Intensive Care Med. 2010, 36:100-106. 10.1007/s00134-009-1634-x

11. Hillman $\mathrm{K}$, Chen J, Cretikos M, et al.: Introduction of the medical emergency team (MET) system: a cluster-randomised controlled trial. Lancet. 2005, 365:2091-2097. 10.1016/S01406736(05)66733-5

12. Kenward G, Castle N, Hodgetts T, Shaikh L: Evaluation of a medical emergency team one year after implementation. Resuscitation. 2004, 61:257-263. 10.1016/j.resuscitation.2004.01.021

13. Chan PS, Jain R, Nallmothu BK, Berg RA, Sasson C: Rapid response teams: a systematic review and meta-analysis. Arch Intern Med. 2010, 170:18-26. 10.1001/archinternmed.2009.424 